\title{
A Discrete Mathematical Modeling and Optimal Control of the Rumor Propagation in Online Social Network
}

\author{
Amine El Bhih $\mathbb{D}^{1},{ }^{1}$ Rachid Ghazzali $\mathbb{D}^{1},{ }^{1}$ Soukaina Ben Rhila $\mathbb{D}^{1},{ }^{1}$ Mostafa Rachik $\mathbb{D}^{1}{ }^{1}$ \\ and Adil El Alami Laaroussi $\mathbb{C}^{1,2}$ \\ ${ }^{1}$ Laboratory of Analysis Modeling and Simulation, Department of Mathematics and Computer Science, \\ Faculty of Sciences Ben M'Sik, Hassan II University Casablanca, BP 7955, Sidi Othman, Casablanca, Morocco \\ ${ }^{2}$ Laboratory of Applied Sciences and Didactics, Higher Normal School Tetouan, Abdelmalek Essaadi University, \\ Tetouan, Morocco \\ Correspondence should be addressed to Amine El Bhih; elbhihamine@gmail.com
}

Received 9 January 2020; Revised 28 March 2020; Accepted 30 March 2020; Published 1 May 2020

Academic Editor: Rodica Luca

Copyright ( $\odot 2020$ Amine El Bhih et al. This is an open access article distributed under the Creative Commons Attribution License, which permits unrestricted use, distribution, and reproduction in any medium, provided the original work is properly cited.

\begin{abstract}
In this paper, a new rumor spreading model in social networks has been investigated. We propose a new version primarily based on the cholera model in order to take into account the expert pages specialized in the dissemination of rumors from an existing IRCSS model. In the second part, we recommend an optimal control strategy to fight against the spread of the rumor, and the study aims at characterizing the three optimal controls which minimize the number of spreader users, fake pages, and corresponding costs; theoretically, we have proved the existence of optimal controls, and we have given a characterization of controls in terms of states and adjoint functions based on a discrete version of Pontryagin's maximum principle. To illustrate the theoretical results obtained, we propose numerical simulations for several scenarios applying the forward-backward sweep method (FBSM) to solve our optimality system in an iterative process.
\end{abstract}

\section{Introduction}

The spread of rumors is a complicated phenomenon that has occupied a large place in human life since ancient times; civilizations have studied and analyzed this phenomenon where many elements overlap and intervene, including what is natural, sociological, economic, and psychological. History has recognized over the years the emergence of many rumors that have spread extensively among communities; it was once the center of interaction and evaluation by company commanders throughout history, and people invent rumors and spread them so that they can be used for political or financial purposes. Rumors can make an industry prosper, while it can also wrest victory from the jaws of defeat. The rumor phenomenon has recognized many changes in its composition with the existence and increasing use of technological capabilities and modern online technologies that companies recognize. This phenomenon has given another dimension since it has been used using the media and genius in the competition between countries and what is recognized as buzz or propaganda and controversy using the publication of false news in whole or partly to influence power with opponents of power. Confined to the elections between Trump and Hillary, where Hillary was the most popular and was the favorite of political researchers [1] until the closing month before the presidential election, where some of the competent communication agencies published many news about Hillary, this news played an important and fundamental role in influencing public opinion and turning the scales in favor of Trump who eventually won, Jennifer et al. in their article [2] gave a contribution to understand the dynamics of this unusual campaign in which social media played a major role. In [3], we can find a collection of Trump tweets divided by subjects (people, places, and things Trump has insulted on twitter) who ultimately received mathematical modeling is one of the most necessary functions of arithmetic that make a contribution to the representation and simulation of social, economic, organic, and ecological phenomena and convert them into mathematical equations that are formulated, studied, analyzed, and interpreted their results as an example; Cazzoli et al. 
analyzed the impact of tweets on the financial market [4], for example, Vilas et al. analyzed the interference between cryptocurrencies and company tickers in the London stock [5].

In this context, many researchers have developed specific mathematical fashions representing the dynamics of the rumor and the factors interfering with its spread [6-9]. In this work and primarily based on the model they proposed, we introduce a new approach by taking into account the spread of rumors through social media. We will describe and explain the model we will use here. As we mentioned, many organizations which specialized in propaganda dissemination have grown to be the usage of social media to facilitate unfold of the rumor and large volume of users. For this purpose, extraordinary pages are created to unfold a rumor about a unique situation or target person. This page is promoted by means of fictitious customers created for this purpose. They create a private network of friends, friends instead are the first victims; each and every time they like or comment on what is posted on the page or fictitious people, this pastime is displayed to all their pals or possibly pals of their buddies inadvertently which is promoted by using this rumor passively by them, while research indicates that the number of users of the networks is rising at a magnificent charge, and it has come to be one of the basics in the discipline of communication and publicity, and in accordance to statistics, what is dealt with millions of rumors spread every day and in view that, in 2019, the entire of Russia is considering the decision to attend some web sites as Telegram because of the threat that brings countrywide security. And if some rumors arouse ridicule, such as announcing that Nicolas Cage was dracula, others were of magnificent danger; to see greater in this regard, we refer the reader to the interesting book [10].

In 1927, Kermack and McKendrick [11] were the first researchers on mathematical epidemiology to suggest the susceptible-infected-removed (SIR) model that describes the rapid explosion of an infectious disease for a short time. In 1964, Goffman and Newill developed in their article titled "Generalization of epidemic principle: an application to the transmission of thoughts," [12] a new notion for modeling the transmission of ideas within a society based on the mathematical model SIR due to the terrific similarity between the two phenomena. This model was earlier used to model the transmission of diseases and epidemics inside communities; in the introduction of their work, the authors cited that the method which has been already described does not take into account the almost endless variety of complexities which in reality arise [12]. Based on the preceding work, Daley and Kendall in their letter titled "Epidemics and Rumours" counseled making use of the preceding thought to modeling the unfold of rumors inside communities [13]. With the development of societies and the emergence of contemporary technological means (transport-communication), new elements have emerged that similarly complicate the phenomenon of rumor and contribute to the massive spread of rumors; this has led many researchers to think about growing the previous model. As an example, the work that has been done by Bettencourt et al. [14], authors proposed a new model taking into account new factors by extending the SIR model to a SEIZR model with two additional compartments. In the same context, Laarabi et al. [15] developed and analyzed the delayed model for the transmission dynamics of rumor with constant recruitment and incubation period. This is in addition to many current works that have currently been produced that take into account quite a few factors concerned in the development of a thought that truly simulates the dynamics of hearsay propagation; to take a broader view, the reader is referred to the article [16]. There are other works which have modeled the phenomenon of rumor propagation using other approaches, for example, complex-network opinion dynamics models, among which, Wang et al. [17] established a new comprehensive model; this model captures the role of memory, the effects of conformity, the differences in the subjective propensity to produce distortions, and the variations in the degree of trust that people have in each other through the concept of entropy of information. In addition, they have developed this model in [18] by taking into account information distortion and polarization effects in order to modeling imprecision in human memory and communication and the consequent progressive drift of information. Based on the single-issue opinion dynamics model, Tan and Cheong [19] incorporated a general model of multi-issue opinion dynamics where the agreement on one issue can promote greater inclusivity in discussing other issues. With the emergence of social networks and their impact on verbal exchange within communities, the place they are taking extra and greater area within the community, it grew to be clear that they should be taken into account as a principal intervening in the unfold of rumors; in this context, many research studies which adopt this hypothesis have been carried out to take thinking on some of these works, see the article [20], for instance, in the work [21], authors had implemented a mathematical model in order to modeling the dynamics of a rumor in the social community with the aid of adding three new compartments: reviewers, sharers, and collectors, people who review the message, accumulate the message, share the message, or do not respond to the message, respectively; however, in the work [21], authors have been restricted to highlight the function of customers of the community and omit the effect of the network itself, in particular, the function of pages that spread the rumor inside the network; the loading of false information in these pages is a source of hearsay between browsers and is considered as a big element which helps in the rapid unfold of rumors, such as rivers and valleys, which store microorganism and microbes and are a hotbed for the multiplication and growth of bacteria that transmit diseases to human beings through the use of water of those rivers; a proper instance of this similarity is the cholera epidemic. In this work, we propose a new model which describes the dynamics of rumor spread through social media based on the cholera model [22] and combine it with the previous work model by adding new cubicles $P$ which represent the page's rumor. Moreover, we apply an optimal control strategy in order to fight against the spread of the rumor through social media; regarding to this, we use theoretical results provided by Balatif et al. [23], where authors implemented a discrete time model that describes the dynamics of voters, and they proposed an optimal control strategy; the same idea and strategy were applied by Labzai et al. [24], and in order to modeling and control smoking, Kouidere et al. [25] suggested a model of the evolution from prediabetes to diabetes 
with optimal control approach. Other models from optimal control problems and population dynamics can be found in [26-30].

In this paper, in Section 2, we propose a discrete $I S_{p} S_{t} P$ mathematical model that describes the dynamic of a population that reacts in the spread of the rumor in a social network. In Section 3, we present an optimal control problem for the proposed model where we give some results concerning the existence of the optimal control, and we characterize the optimal controls using the Pontryagin maximum principle in discrete time. Numerical simulations through MATLAB are given in Section 4. Finally, we conclude the paper in Section 5.

\section{Mathematical Model and Simulation without Controls}

In this section, we consider a discrete mathematical model $I S_{p} S_{t} P$ that will describe the dynamics of a population of rumors; our model consists of four compartments representing the subdivision of the population that reacts in the spread of the rumor in a social network:

I: ignorant, users who do not know the rumor and susceptible to be informed

$S_{p}$ : spreader, users who spread the rumor

$S_{t}$ : stifler, individuals who refuse to spread the rumor

$P$ : rumor's page, the page specialized in spreading the rumor

The compartment $I$ represents the number of users who do not understand the rumor and who are susceptible to be informed, and this population increases with the charge $\mu \mathrm{N}$ which represents the new customers created; an ignorant inquires about the rumor through two ways, either by using consulting a specialized page in the diffusion of the rumor or at once by means of the contact with a spreader; some of these users deactivate their account at a rate $\mu I$. Thus, in this compartment, we have an incoming flux which equals to $\mu N$ and an outgoing flux which equals to $\alpha_{h} S_{p} I+\alpha_{e} I(P / \kappa+P)+\mu I$.

The compartment $S_{p}$ : this compartment represents the number of humans who spread the rumor either immediately or by way of sharing one-page publications or via developing new publications. Thus, we have an incoming flux which equals to $\theta\left(\alpha_{h} S_{p} I+\alpha_{e} I(P / \kappa+P)\right)$ which represents the proportion of the new users who will spread the rumor. After the contact between two spreaders, one of them decides not to diffuse the information at a rate $\gamma S_{p}^{2}$, and after the contact of a spreader and a stifler, the stifler succeeds to convince him that the information is false at a rate $\lambda S_{t} S_{p}$; after a certain period, a portion of the spreaders decide not to spread the rumor at a rate $\beta S_{p}$.

The compartment $S_{t}$ : this compartment represents the number of stiflers who refuse to spread the rumor. This number increases at a rate $(1-\theta)\left(\alpha_{h} S_{p} I+\alpha_{e} I(P / \kappa+P)\right)$ which represents the portion of users who knew that the information is wrong, in addition to the flux that left the $S_{p}$ compartment $S_{p}\left(\gamma S_{p}+\lambda S_{t}\right)+\beta S_{p}$, and decreases with the rate $\mu_{1} S_{t}$ of stiflers who have deactivated their accounts.

The compartment $P$ : this compartment represents the page specialized in the diffusion of the rumor. This page consists of malicious publications about the rumor; in this page, $S_{p}$ has the proper right to publish and share these publications at a rate $\delta_{1} S_{p}$ and $\varepsilon_{2} S_{p}$, respectively, and the ignorant who consults the page also shares these publications at a rate $\varepsilon_{1} I$.

The diagram will demonstrate the flux directions of individuals among the compartments, Figure 1.

$$
\left\{\begin{array}{l}
I(n+1)=I(n)+\mu N-\alpha_{h} S_{p}(n) I(n)-\alpha_{e} I(n) \frac{P(n)}{\kappa+P(n)}-\mu_{1} I(n), \\
S_{p}(n+1)=S_{p}(n)+\theta\left(\alpha_{h} S_{p}(n) I(n)+\alpha_{e} I(n) \frac{P(n)}{\kappa+P(n)}\right)-\left(\mu_{1}+\beta\right) S_{p}(n)-\gamma S_{p}(n)\left(S_{p}(n)+S_{t}(n)\right), \\
S_{t}(n+1)=S_{t}(n)+(1-\theta)\left(\alpha_{h} S_{p}(n) I(n)+\alpha_{e} I(n) \frac{P(n)}{\kappa+P(n)}\right)+\gamma S_{p}(n)\left(S_{p}(n)+S_{t}(n)\right)+\beta S_{p}(n)-\mu_{1} S_{t}(n), \\
P(n+1)=P(n)+\varepsilon_{1} N+\varepsilon_{2} S_{p}(n)-\mu_{2} P(n),
\end{array}\right.
$$

with initial values $I(0), S_{p}(0), S_{t}(0)$, and $P(0)$ which are nonnegatives.

In order to demonstrate the effectiveness of the model we have proposed, we will present a numerical simulation with Figure 2 so that we can see how well the model adapts to reality. Initial values are approximate data that we suggested after studying and researching some statistics about the users of social networks, and the values are attached in the table.
From Figure 2, we note that there is no significant effect until the 30th day; 30 days after the launch of the rumor, the number of ignorants decreases sharply, and in contrast, there is a significant rise of spreader people and the number of stiflers and pages are rising on average; these changes indicate that, after 30 days, trading rumor has become more and more due to the continuous publication of it. 


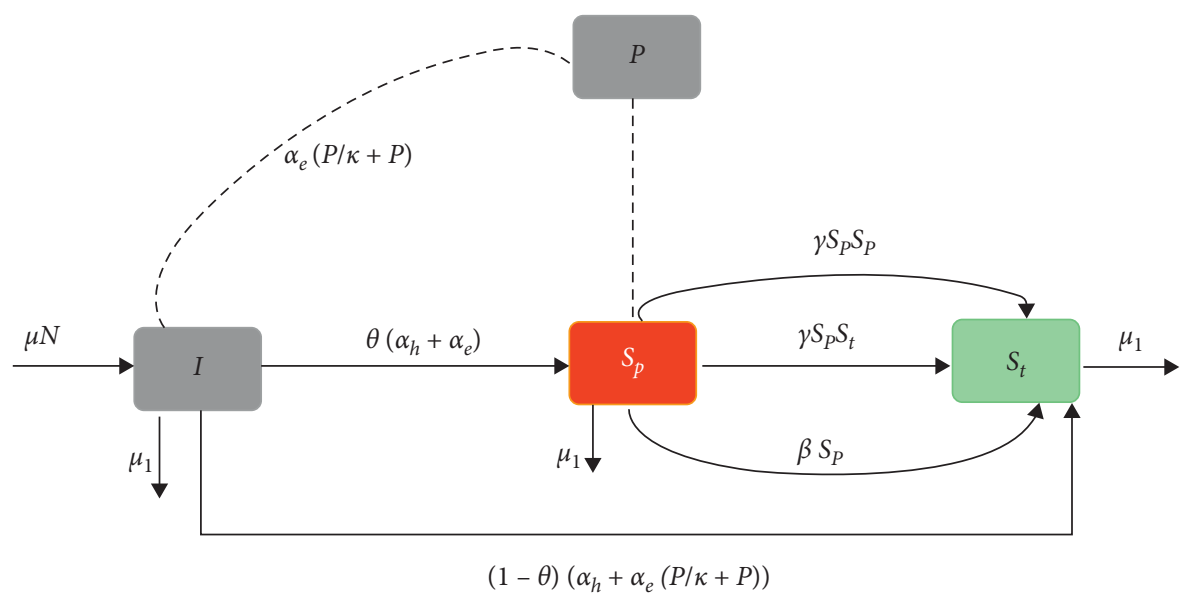

FIgURe 1: Description diagram of the rumor dynamics.

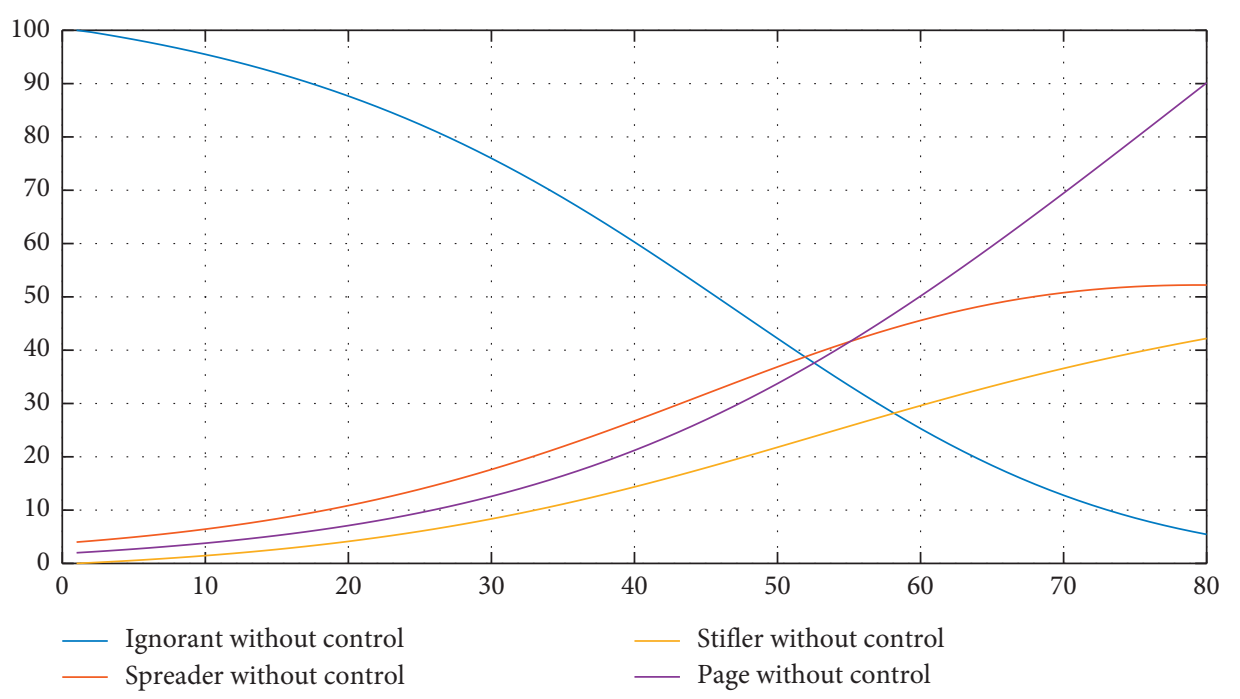

Figure 2: Dynamics without control strategy.

\section{The Model with Controls}

Now, we introduce our controls into system (1) as the control measures to fight the spread of the rumor; we extend our system by including three kinds of controls $u, v$, and $w$. The first control $u$ is to tell users that the information or publication is false and contains a malicious rumor, the second control $v$ is through the admin where he deactivates an account after learning that it is fake or aimed at spreading the rumor, and the last one $w$ is also applied by the admin, this time by deactivating the page intended to spread the rumor after the arrival of a certain number of complaints.

In the aim of better understanding the effects of any control measure of these strategies, we introduce three new variables: $\pi_{i}$, where $i=1,2,3, \pi_{i}=0$ in the absence of the control, and $\pi_{i}=1$ in the presence of the control.

$$
\left\{\begin{array}{l}
I(n+1)=I(n)+\mu N-\alpha_{h} S_{p}(n) I(n)-\alpha_{e} I(n) \frac{P(n)}{\mathcal{\kappa}+P(n)}-\mu_{1} I(n)-\pi_{1} u_{n} I(n), \\
S_{p}(n+1)=S_{p}(n)+\theta\left(\alpha_{h} S_{p}(n) I(n)+\alpha_{e} I(n) \frac{P(n)}{\kappa+P(n)}\right)-\left(\mu_{1}+\beta\right) S_{p}(n)-\gamma S_{p}(n)\left(S_{p}(n)+S_{t}(n)\right)-\pi_{2} v_{n} S_{p}(n), \\
S_{t}(n+1)=S_{t}(n)+(1-\theta)\left(\alpha_{h} S_{p}(n) I(n)+\alpha_{e} I_{n} \frac{P_{n}}{\mathcal{\kappa}+P_{n}}\right)+\gamma S_{p}(n)\left(S_{p}(n)+S_{t}(n)\right)+\beta S_{p}(n)-\mu_{1} S_{t}(n)+\pi_{1} u_{n} I_{n}, \\
P(n+1)=P_{n}+\varepsilon_{1} N+\varepsilon_{2} S_{p}(n)-\mu_{2} P_{n}-\pi_{3} w_{n} P_{n} .
\end{array}\right.
$$




\section{An Optimal Control Approach}

The problem is to minimize the objective functional:

$$
\begin{aligned}
J(u, v, w)= & M_{N} S_{p}(N)+\sum_{k=0}^{N-1}\left(M_{k} S_{p}(k)+\frac{1}{2} A_{k} \pi_{1} u_{k}^{2}\right. \\
& \left.+\frac{1}{2} B_{k} \pi_{2} v_{k}^{2}+\frac{1}{2} C_{k} \pi_{3} w_{k}^{2}\right),
\end{aligned}
$$

where $A_{k}>0, B_{k}>0, C_{k}>0$, and $M_{k}>0$ for $i \in\{0, \ldots, N\}$ are the cost coefficients. They are selected to weigh the relative importance of $S_{p}(k), u_{k}, v_{k}$, and $w_{k}$ at time $k$. Nis the final time.

In other words, we seek the optimal controls $u_{k}, v_{k}$, and $w_{k}$ such that

$$
J\left(u^{*}, v^{*}, w^{*}\right)=\min _{(u, v, w)}\left\{\frac{J(u, v, w)}{(u, v, w)} \in U_{\text {ad }}\right\},
$$

where $U_{\text {ad }}$ is the set of admissible controls defined by

$$
U_{\mathrm{ad}}=\left\{\frac{\left(u_{k}, v_{k}, w_{k}\right)}{a \leq u_{k} \leq b, c \leq v_{k} \leq d, e \leq w_{k} \leq f}\right\}, \quad k=\{0, \ldots, N-1\},
$$

and $(a, b, c, d, e, f) \in(] 0,1[)^{6}$.

The sufficient condition for the existence of an optimal control $\left(u_{k}^{*}, v_{k}^{*}, w_{k}^{*}\right)$ for problem (3) comes from the following theorem.

Theorem 1. There exists an optimal control $\left(u^{*}, v^{*}, w^{*}\right)$ such that

$$
J\left(u^{*}, v^{*}, w^{*}\right)=\min _{(u, v, w)}\left\{\frac{J(u, v, w)}{(u, v, w)} \in U_{\mathrm{ad}}\right\},
$$

subject to control system (2) with initial conditions.

Proof. There are a finite number of time steps, $S=$ $\left(S_{0} ; S_{1} ; \ldots ; S_{n}\right), I=\left(I_{0} ; I_{1} ; \ldots ; I_{N}\right)$, and $P=\left(P_{0} ; P_{1} ; \ldots\right.$;
$\left.P_{N}\right)$ which are uniformly bounded for all $(u, v, w) \in U_{\text {ad }}$. Thus, $J(u, v, w)$ is uniformly bounded for all $(u, v, w)$ in the control set $U_{\mathrm{ad}}$. Since $J(u, v, w)$ is bounded,inf ${ }_{(u, v, w) \in U_{\mathrm{ad}}} J(u, v, w)$ is finite, and there exists a sequence $u_{k}^{j} ; v_{k}^{j} ; w_{k}^{j} \in U_{\text {ad }}$ such that $\lim _{j \longrightarrow+\infty} J\left(u_{k}^{j} ; v_{k}^{j} ; w_{k}^{j}\right)=$ $\inf _{(u, v, w) \in U_{\mathrm{ad}}} J(u, v, w)$ and the corresponding sequences of states $I^{i}, S_{t}^{i}, S_{p}^{i}$, and $B^{i}$. Since there is a finite number of uniformly bounded sequences, there exist $\left(u_{k}^{*} ; v_{k}^{*} ; w_{k}^{*}\right) \in U_{\text {ad }}^{3}$ and $S, I, P, R \in \mathbb{R}^{N+1}$ such that, on a subsequence, $u_{k}^{j} \longrightarrow u_{k}^{*}, v_{k}^{j} \longrightarrow v_{k}^{*}, w_{k}^{j} \longrightarrow w_{k}^{*}, S^{j} \longrightarrow S^{*}$, $I^{j} \longrightarrow I^{*}$, and $P^{j} \longrightarrow P^{*}$. Finally, due to the finite dimensional structure of system (2) and the objective function $J(u, v, w),\left(u^{*}, v^{*}, w^{*}\right)$ is an optimal control with corresponding states $I^{*}, S_{t}^{*}, S_{p}^{*} P^{*}$ Therefore, $\inf _{(u, v, w) \in U_{\mathrm{ad}}} J(u, v, w)$ is achieved.

In order to derive the necessary condition for the optimal control, Pontryagin's maximum principle in discrete time given in [31, 32] was used. This principle converts into a problem of minimizing a Hamiltonian at the time step defined by

$$
\begin{aligned}
H_{k}= & M_{k} S_{p}(k)+\frac{1}{2} A_{k} \pi_{1} u_{k}^{2}+\frac{1}{2} B_{k} \pi_{2} v_{k}^{2} \\
& +\frac{1}{2} C_{k} \pi_{3} w_{k}^{2}+\sum_{j=1}^{4} \lambda_{j, k+1} f_{j, k+1},
\end{aligned}
$$

where $f_{j, k+1}$ is the right side of the system of difference equation (2) of the $j^{\text {th }}$ state variable at time step $k+1$.

Using Pontryagin's maximum principle in discrete time [31-33], we can say the following theorem.

Theorem 2. Let $I^{*}, S_{p}^{*}, S_{t}^{*}$, and $P^{*}$ be optimal state solutions with an associated optimal control $\left(u^{*}, v^{*}, w^{*}\right)$ for optimal control problem (3). Then, there exist adjoint variables $\lambda_{1, k}, \lambda_{2, k}, \lambda_{3, k}, \lambda_{4, k}$ satisfying

$$
\left\{\begin{array}{l}
\Delta \lambda_{1, k}=\lambda_{1, k+1}\left[1-\alpha_{h} S_{p}(k)-\alpha_{e} \frac{P(k)}{\kappa+P(k)}-\mu_{1}-\pi_{1} u_{k}\right]+\lambda_{2, k+1} \theta\left[\alpha_{h} S_{p}(k)+\alpha_{e} \frac{P(k)}{\kappa+P(k)}\right] \\
\quad+\lambda_{3, k+1}(1-\theta)\left[\alpha_{h} S_{p}(k)+\alpha_{e} \frac{P(k)}{\kappa+P(k)}\right] \\
\Delta \lambda_{2, k}=1+\lambda_{1, k+1}\left[-\alpha_{h} I(k)\right]+\lambda_{2, k+1}\left[1+\theta \alpha_{h} I(k)-(\mu+\beta)-2 \gamma-\pi_{2} v_{k}(k)\right]+\lambda_{3, k+1}\left[(1-\theta)\left(\alpha_{h} S_{p}(k)\right)+2 \gamma+\beta\right]+\lambda_{4, k+1} \varepsilon_{2}, \\
\Delta \lambda_{3, k}=\lambda_{2, k+1}\left[-\gamma S_{p}(k) S_{p}(k)\right]+\lambda_{3, k+1}\left[1+\gamma S_{p}(k)-\mu\right], \\
\Delta \lambda_{4, k}=\lambda_{1, k+1}\left[-\alpha_{e} I(k) \frac{\kappa}{(\kappa+P(k))^{2}}\right]+\lambda_{2, k+1} \theta\left[\alpha_{e} I(k) \frac{\kappa}{(\kappa+P(k))^{2}}\right]+\lambda_{3, k+1}(1-\theta)\left[\alpha_{e} I(k) \frac{\kappa}{(\kappa+P(k))^{2}}\right] \\
\quad+\lambda_{4, k+1}\left(1-\mu_{2}-\pi_{3} w_{k}\right),
\end{array}\right.
$$


with transversality conditions at time

$$
\begin{aligned}
& \lambda_{1, N}=A_{N}, \\
& \lambda_{2, N}=\lambda_{3, N}=\lambda_{4, N}=0 .
\end{aligned}
$$

Furthermore, for $k=0,1, \ldots, N-1$ and for $\pi_{1}=\pi_{2}=\pi_{3}=1$, the optimal controls $u_{k}^{*}, v_{k}^{*}$, and $w_{k}^{*}$ are given by

$$
\begin{aligned}
& u_{k}^{*}=\min \left(b, \max \left(a, \frac{\lambda_{1, k+1} I(k)-\lambda_{3, k+1} \gamma S_{p}(k) I(k)}{A_{k}}\right)\right), \\
& v_{k}^{*}=\min \left(d, \max \left(c, \frac{-\lambda_{2, k+1} \gamma\left(S_{p}(k)\right)^{2} S_{t}(k)}{B_{k}}\right)\right), \\
& w_{k}^{*}=\min \left(f, \max \left(e, \frac{\lambda_{4, k+1} P(k)}{C_{k}}\right)\right) .
\end{aligned}
$$

Proof. The Hamiltonian of the optimal problem is given by

$$
\begin{aligned}
H_{k}= & M_{k} S_{p}(k)+\frac{1}{2} A_{k} u_{k}^{2}+\frac{1}{2} B_{k} v_{k}^{2}+\frac{1}{2} C_{k} w_{k}^{2}+\lambda_{1, k+1}\left(I(k)+\mu N-\alpha_{h} S_{p}(k) I(k)-\alpha_{e} I(k) \frac{P(k)}{\kappa+P(k)}-\mu_{1} I(k)-\pi_{1} u_{k} I\right) \\
& +\lambda_{2, k+1}\left(S_{p}(k)+\theta\left(\alpha_{h} S_{p}(k) I(k)+\alpha_{e} I(k) \frac{P(k)}{\kappa+P(k)}\right)-(\mu+\beta) S_{p}(k)-\gamma S_{p}(k)\left(S_{p}(k)+S_{t}(k)\right)-\pi_{2} v_{k} S_{p}(k)\right) \\
& +\lambda_{3, k+1}\left(S_{t}(k)+(1-\theta)\left(\alpha_{h} S_{p}(k) I(k)+\alpha_{e} I(k) \frac{P(k)}{\kappa+P(k)}\right)+\gamma S_{p}(k)\left(S_{p}(k)+S_{t}(k)\right)+\beta S_{p}(k)-\mu S_{t}(k)+\pi_{1} u_{k} I\right) \\
& +\lambda_{4, k+1}\left(P(k)+\varepsilon_{1} N(k)+\varepsilon_{2} S_{p}(k)-\mu_{2} P(k)-\pi_{3} w_{k} P(k)\right) .
\end{aligned}
$$

For $k=0,1, \ldots, N-1$, the adjoint equations and transversality conditions can be obtained by using

Pontryagin's maximum principle, in discrete time, given in [31-33] such that

$$
\left\{\begin{array}{l}
\Delta \lambda_{1, k}=\frac{\partial H_{k}}{\partial I_{k}}=\lambda_{1, k+1}\left[1-\alpha_{h} S_{p}(k)-\alpha_{e} \frac{P(k)}{\kappa+P(k)}-\mu_{1}-\pi_{1} u_{k}\right]+\lambda_{2, k+1} \theta\left[\alpha_{h} S_{p}(k)+\alpha_{e} \frac{P(k)}{\kappa+P(k)}\right] \\
+\lambda_{3, k+1}(1-\theta)\left[\alpha_{h} S_{p}(k)+\alpha_{e} \frac{P(k)}{\kappa+P(k)}\right], \\
\Delta \lambda_{2, k}=\frac{\partial H_{k}}{\partial S_{p}(k)}=M_{k}+\lambda_{1, k+1}\left[-\alpha_{h} I(k)\right]+\lambda_{2, k+1}\left[1+\theta \alpha_{h} I(k)-(\mu+\beta)-2 \gamma-\pi_{2} v_{k}\right] \\
\quad+\lambda_{3, k+1}\left[(1-\theta)\left(\alpha_{h} S_{p}(k)\right)+2 \gamma+\beta\right]+\lambda_{4, k+1} \varepsilon_{2}, \\
\Delta \lambda_{3, k}=\frac{\partial H_{k}}{\partial S_{t}(k)}=\lambda_{2, k+1}\left[-\gamma S_{p}(k)\right]+\lambda_{3, k+1}\left[1+\gamma S_{p}(k)-\mu\right], \\
\Delta \lambda_{4, k}=\frac{\partial H_{k}}{\partial P_{k}}=\lambda_{1, k+1}\left[-\alpha_{e} I(k) \frac{\kappa}{(\kappa+P(k))^{2}}\right]+\lambda_{2, k+1} \theta\left[\alpha_{e} I(k) \frac{\kappa}{(\kappa+P(k))^{2}}\right]+\lambda_{3, k+1}(1-\theta)\left[\alpha_{e} I(k) \frac{\kappa}{(\kappa+P(k))^{2}}\right] \\
\quad+\lambda_{4, k+1}\left(1-\mu_{2}-\pi_{3} w_{k}\right) .
\end{array}\right.
$$


Put $\Phi(N)=A_{N} I_{N}$ :

$$
\begin{aligned}
& \lambda_{1, N}=\frac{\partial \Phi(N)}{\partial I}=A_{N}, \\
& \lambda_{2, N}=\frac{\partial \Phi(N)}{\partial S_{p}}=0, \\
& \lambda_{3, N}=\frac{\partial \Phi(N)}{\partial S_{t}}=0, \\
& \lambda_{4, N}=\frac{\partial \Phi(N)}{\partial P}=0 .
\end{aligned}
$$

For $k=0,1, \ldots, N-1$, the optimal controls $u_{k}^{*}, v_{k}^{*}$, and $w_{k}^{*}$ can be solved from the following optimality condition:

$$
\begin{aligned}
\frac{\partial H}{\partial u_{k}} & =A_{k} u_{k}-\lambda_{1, k+1} \pi_{1} I(k)+\lambda_{3, k+1} \gamma \pi_{1} S_{p}(k) I(k)=0 \Longleftrightarrow \\
u_{k} & =\frac{\lambda_{1, k+1} \pi_{1} I(k)-\lambda_{3, k+1} \gamma \pi_{1} S_{p}(k) I(k)}{A_{k}}, \\
\frac{\partial H}{\partial v_{k}} & =B_{k} v_{k}+\lambda_{2, k+1} \gamma\left(S_{p}(k)\right)^{2} \pi_{2}(k)=0 \Longleftrightarrow \\
v_{k} & =\frac{-\lambda_{2, k+1} \gamma\left(S_{p}(k)\right)^{2} \pi_{2} S_{t}(k)}{B_{k}}, \\
\frac{\partial H}{\partial w_{k}} & =C_{k} w_{k}-\lambda_{4, k+1} \pi_{3} P(k)=0 \Longleftrightarrow \\
w_{k} & =\frac{\lambda_{4, k+1} \pi_{3} P(k)}{C_{k}} .
\end{aligned}
$$

So, for $\pi_{1}=\pi_{2}=\pi_{3}=1$, we have

$$
\begin{aligned}
& u_{k}=\frac{\lambda_{1, k+1} I(k)-\lambda_{3, k+1} \gamma S_{p}(k) I(k)}{A_{k}}, \\
& v_{k}=\frac{-\lambda_{2, k+1} \gamma\left(S_{p}(k)\right)^{2} S_{t}(k)}{B_{k}}, \\
& w_{k}=\frac{\lambda_{4, k+1} P(k)}{C_{k}} .
\end{aligned}
$$

However, if $\pi_{i}=0$ for $i=1,2,3$, the control attached to this case will be eliminated and removed. By the bounds in $U_{\text {ad }}$ of the controls, it is easy to obtain $u_{k}^{*}, v_{k}^{*}$, and $w_{k}^{*}$ in the form of (10).

4.1. Algorithm. In this section, we present the results obtained by solving numerically the optimality system. This system consists of the state system, adjoint system, initial and final time conditions, and the control characterization. So, the optimality system is given by the following:

Step 1: $I_{0}=i_{0}, \quad S_{p 0}=s_{p 0}, \quad S_{t 0}=s_{t 0}, \quad P_{0}=p_{0}$, $\lambda_{2, N}=\lambda_{3, N}=\lambda_{4, N}=0, \lambda_{1, N}=A_{N}$, and given $u_{k ; 0}^{*}, v_{k ; 0}^{*}$, and $w_{k ; 0}^{*}$

Step 2: for $k=0 ; 1 ; \ldots ; N-1$ do:

$$
\begin{aligned}
I_{k+1} & =\mu N-\alpha_{h} S_{p k} I_{k}-\alpha_{e} I_{k} \frac{P_{k}}{\kappa+P_{k}}-\mu_{1} I_{k}, \\
S_{p(k+1)} & =\theta\left(\alpha_{h} S_{p k} I_{k}+\alpha_{e} I_{k} \frac{P_{k}}{\kappa+P_{k}}\right)-(\mu+\beta) S_{p k}-\gamma S_{p k}\left(S_{p k}+S_{t k}\right), \\
S_{p(k+1)} & =(1-\theta)\left(\alpha_{h} S_{p k} I_{k}+\alpha_{e} I_{k} \frac{P_{k}}{\kappa+P_{k}}\right)+\gamma S_{p k}\left(S_{p k}+S_{t k}\right)+\beta S_{p k}-\mu S_{t k}, \\
P_{k+1} & =\varepsilon_{1} N+\varepsilon_{2} S_{p k}-\mu_{2} P_{k}, \\
\quad \vdots & \vdots \\
\vdots & \\
\lambda_{1, T-k} & =\lambda_{1, T-k+1}\left(1-\alpha_{h} S_{p k}-\alpha_{e} \frac{P_{k}}{\kappa+P_{k}}-\mu_{1}\right)+\lambda_{2, T-k+1} \theta\left(\alpha_{h} S_{p k}+\alpha_{e} \frac{P_{k}}{\kappa+P_{k}}\right)+\lambda_{3, T-k+1}(1-\theta)\left(\alpha_{h} S_{p k}+\alpha_{e} \frac{P_{k}}{\kappa+P_{k}}\right), \\
\lambda_{2, T-k} & =1+\lambda_{1, T-k+1}\left(-\alpha_{h} I_{k}\right)+\lambda_{2, T-k+1}\left(1+\theta \alpha_{h} I_{k}-(\mu+\beta)-2 \gamma\right)+\lambda_{3, T-k+1}\left[(1-\theta)\left(\alpha_{h} S_{p k}\right)+2 \gamma+\beta\right]+\lambda_{4, T-k+1} \varepsilon_{2},
\end{aligned}
$$




$$
\begin{aligned}
\lambda_{3, T-k} & =\lambda_{2, T-k+1}\left[-\gamma S_{p k} S_{p k}\right]+\lambda_{3, T-k+1}\left[1+\gamma S_{p k}-\mu\right] \\
\lambda_{4, T-k} & =\lambda_{1, T-k+1}\left[-\alpha_{e} I_{k} \frac{\kappa}{\left(\kappa+P_{k}\right)^{2}}\right]+\lambda_{2, T-k+1} \theta\left[\alpha_{e} I_{k} \frac{\kappa}{\left(\kappa+P_{k}\right)^{2}}\right]+\lambda_{3, T-k+1}(1-\theta)\left[\alpha_{e} I_{k} \frac{\kappa}{\left(\kappa+P_{k}\right)^{2}}\right]+\lambda_{4, T-k+1}\left(1-\mu_{2}\right) \\
u_{k+1} & =\min \left[b, \max \left(a, \frac{1}{A_{k}}\left(\lambda_{1, T-k+1} I_{k}-\lambda_{3, T-k+1} \gamma S_{p k} I_{k}\right)\right)\right] \\
v_{k+1} & =\min \left[d, \max \left(c, \frac{1}{B_{k}}\left(-\lambda_{2, T-k+1} \gamma\left(S_{p k}\right)^{2} S_{t k}\right)\right)\right] \\
w_{k+1} & =\min \left[f, \max \left(e, \frac{1}{C_{k}}\left(-\lambda_{4, T-k+1} P_{k}\right)\right)\right]
\end{aligned}
$$

end for

Step 3: for $k=0 ; 1 ; \ldots ; N$; write:

$$
\begin{gathered}
I_{k}^{*}=I_{k}, \\
S_{p k}^{*}=S_{p k}, \\
S_{t k}^{*}=S_{t k}, \\
P_{k}^{*}=P_{k}, \\
u_{k}^{*}=u_{k}, \\
v_{k}^{*}=v_{k}, \\
w_{k}^{*}=w_{k},
\end{gathered}
$$

end for.

In this formulation, there were initial conditions for the state variables and terminal conditions for the adjoints. That is, the optimality system is a two-point boundary value problem with separated boundary conditions at time steps $k=0$ and $k=N$. We solve the optimality system by an iterative method with forward solving of the state system followed by backward solving of the adjoint system. We start with an initial guess for the controls at the first iteration, and then before the next iteration, we update the controls by using the characterization. We continue until convergence of successive iterates is achieved.

\section{Numerical Simulation}

In this paragraph, we give numerical simulation to highlight the effectiveness of the strategy that we have developed in the framework of eliminating the rumor and limit its spread, and the initial values are the same as in Table 1; with regard to other initial values, they are proposed values after a statistical study.

In this section, we introduce our control strategy which consists in using three kinds of controls: the first one $u_{n}$ where an ignorant is informed by a stifler that the information is false or a rumor, the second noted $v_{n}$ which is applied by a professional admin and which consists of deactivating or blocking a fake account that specializes in spreading the rumor, and the third noted $w_{n}$ which is also applied by an admin by blocking the pages created in order to spread the rumor and this after reaching a significant number of signals and after the diagnosis of the content of the concerned page. We apply our strategy for a period of fifteen weeks that we assume an average duration of the spread of a rumor. Figure 3 illustrates the results found. From Figure 3, we see that the effect of the strategy begins as early as the second week, and the number of the ignorant decreases from 2000 in a fatal way until reaching almost 100; this number is recovered in the number of stiflers which increases significantly from zero until reaching 2000. The same remark for the number of pages, we notice that between the first and the second week, the number increases step by step; however, after the second week of the application of controls, the number decreases until less than 50 . As for the number of spreaders, the number is decreasing from 50 to zero.

In the next section and in order to give more details about our strategy as well as the effect of each control, we choose to apply three scenarios; in each of these scenarios, we apply separately one control, Figures 4-6 illustrate the result found in each scenario for the three cases:
(a) Apply only the control $u$
(b) Apply only the control $v$
(c) Apply only the control $w$

5.1. Apply Only the Control $u$. In this scenario, we simulate the case where we apply a single control $u$ with which we inform a portion of the ignorants by the false information, so we win this proportion in favor of stiflers, and the control is applied over a period of 100 days. From the figure, we see that, after 10 days, the number of spreaders falls and does not exceed 3,000 people throughout the period, and the number of stiflers meanwhile increases sharply to almost 20,000 which shows the effectiveness of our control.

5.2. Apply Only the Control v. In the second scenario, we apply a single control $v$ but this time, a control that focuses 
TABLE 1: Rumor model parameters and values.

\begin{tabular}{lcc}
\hline Parameter & Description & Value \\
\hline$\alpha_{h}$ & The proportion of ignorants who become the spreader after discussing with a spreader & $(0.0005 /$ day) \\
$\alpha_{e}$ & The proportion of ignorants who become the spreader after consulting a page of rumor & $(0.0007 /$ day) \\
$\theta$ & The proportion of the ignorant who become the spreader & $(0.65 /$ day) \\
$(1-\theta)$ & The proportion of the ignorant who become the stifler & $(0.35 /$ day) \\
$\mu$ & New users and deactivated users & $0.0008 /$ day \\
$\beta$ & The proportion of spreaders who become stiflers & $(0.002 /$ day) \\
$\gamma$ & The proportion of spreaders who become stiflers after contacting another spreader & $(0.00005 /$ day) \\
$\varepsilon_{1}$ & The rate of shared publications of a rumor page by ignorants & $(0.000005 /$ day) \\
$\varepsilon_{2}$ & The rate of shared publications of a rumor page by spreaders & $0.00001 /$ day \\
$\delta_{1}$ & New rumor pages created by spreaders & $0.04 /$ day \\
\hline
\end{tabular}

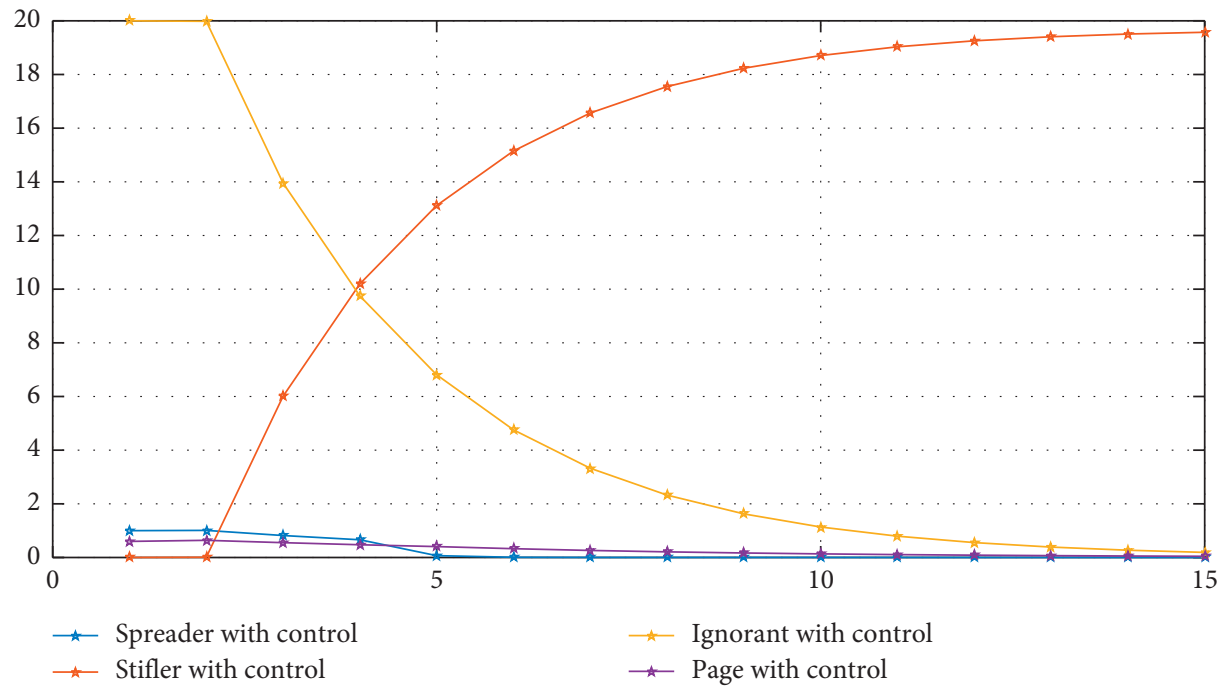

FIGURe 3: Dynamics with controls $u, v$, and $w$.

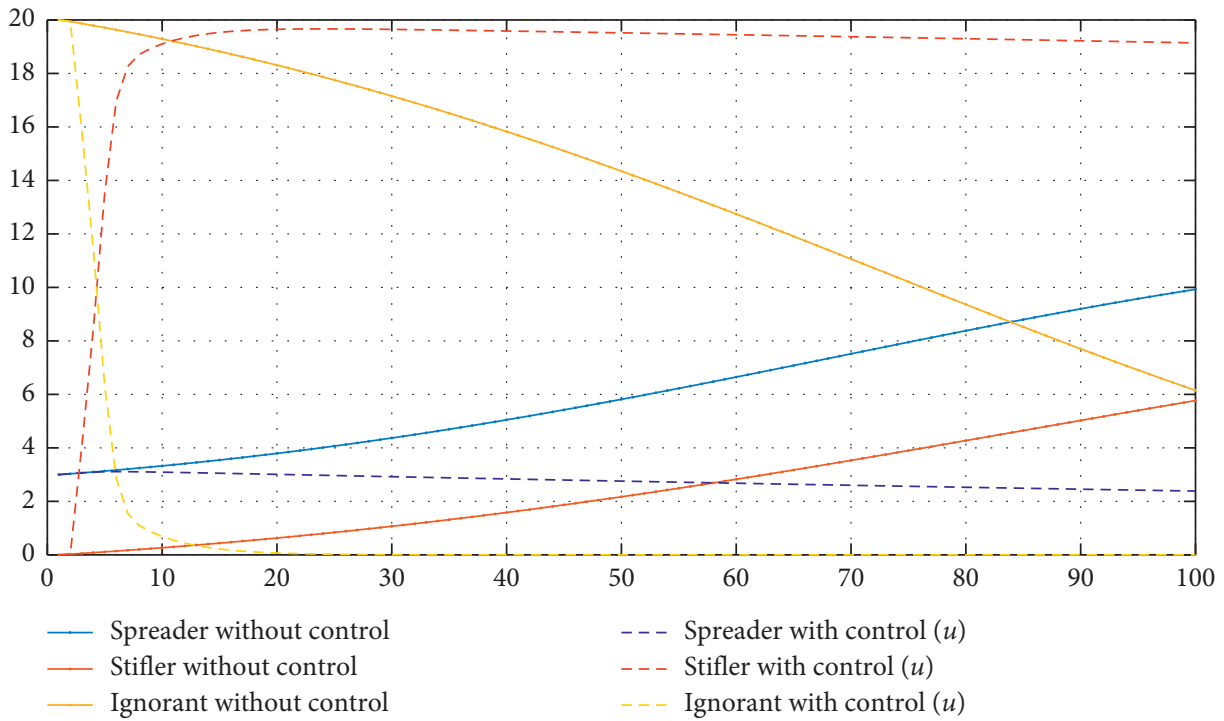

Figure 4: Dynamics with control $u$.

on the spreaders by disabling their accounts, and the period is always 100 days; the figure shows us that the number of spreaders decreases since the 3rd day, but this time, we see that the number Sp tends to zero; however, there is a small improvement in the number of stiflers, and we notice that the number grows slowly and reaches 10,000 people. 


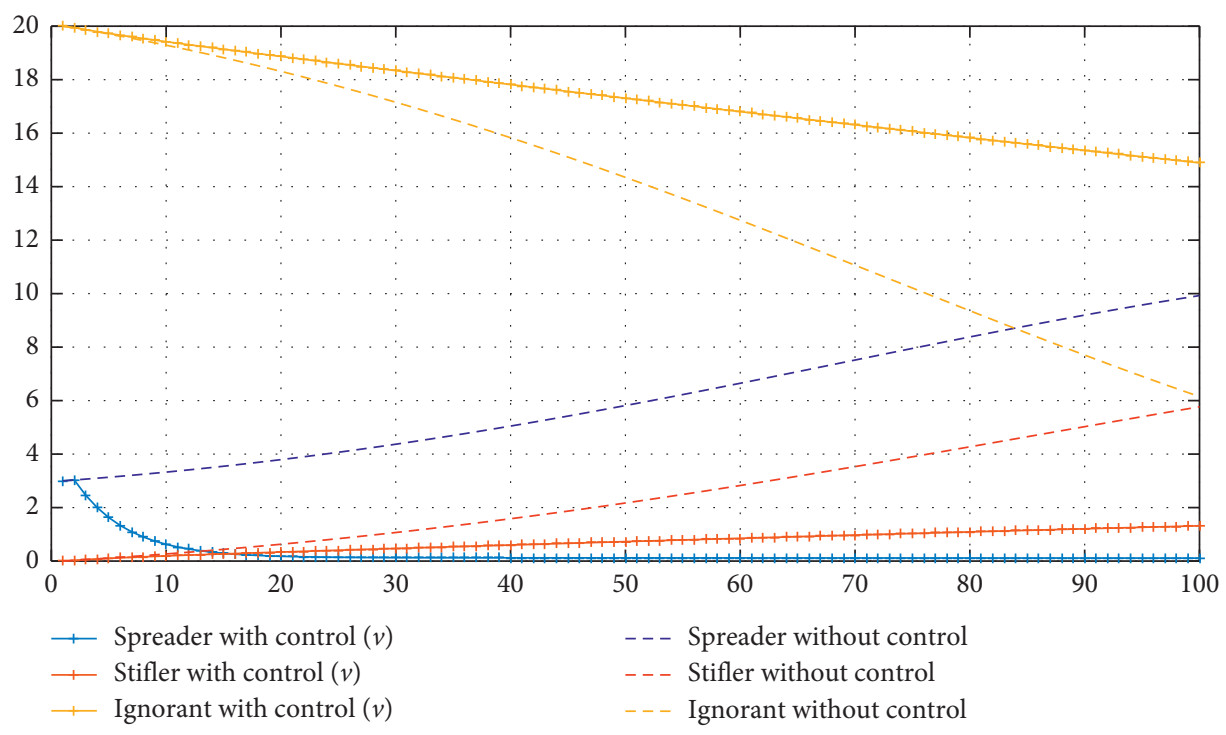

Figure 5: Dynamics with control $v$.

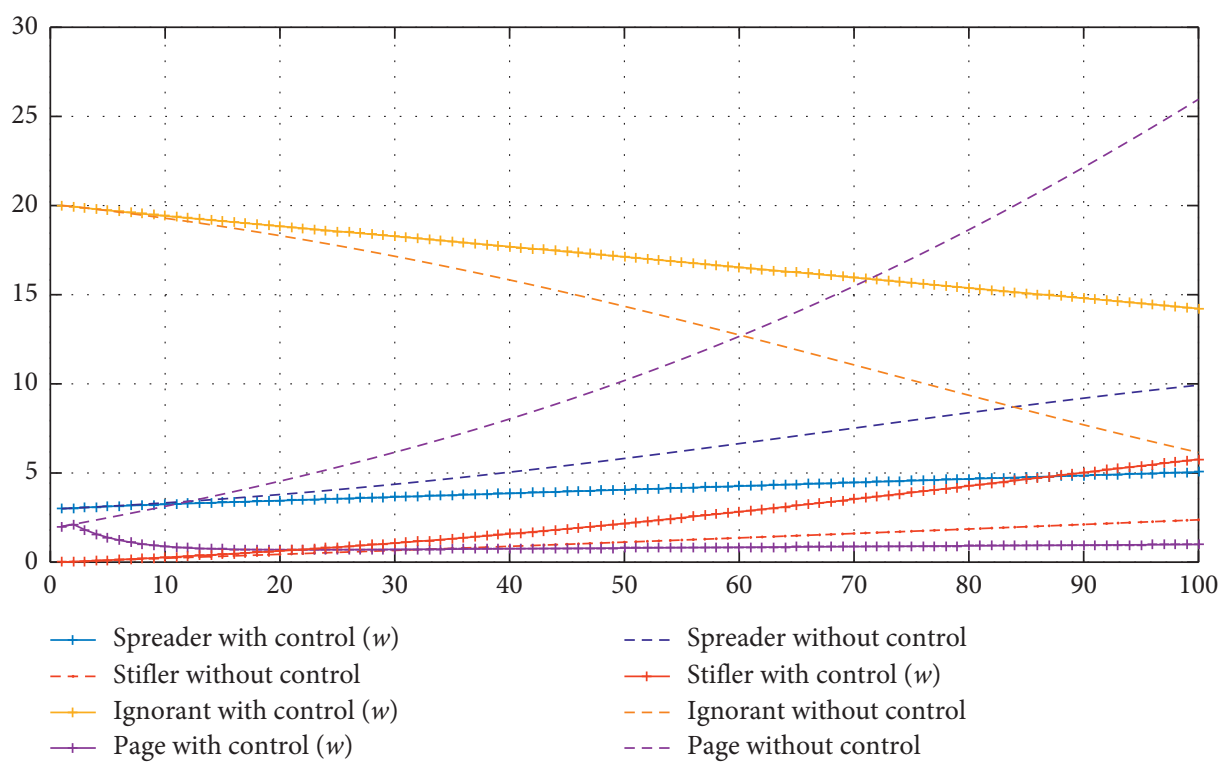

Figure 6: Dynamics with control $w$.

5.3. Apply Only the Control $w$. In the last scenario, we apply the only remaining control $w$ by disabling the pages reserved for the diffusion of the rumor. From the figure, we see that the numbers of stiflers and spreaders have undergone the same changes as in the previous scenarios, and the number of pages has fallen dramatically, which shows the effectiveness of this strategy because it gives similar results to the previous ones and more, which is logical since this strategy aims and attacks the source of the rumor and the necessary tool for spreaders in order to spread the rumor.

\section{Conclusion}

In this work, we propose a new model which describes the dynamics of rumor spread through social media based on the cholera model; this allows us to introduce a new compartment representing pages or tweets or any other kind of publication that disseminates the rumor. Moreover, we apply a control strategy in order to fight against the spread of the rumor; since there is an overlap between both phenomena, the strategy is also based on the cholera model and which proved its effectiveness against this disease. Three control strategies were introduced, and by the introduction of three new variables $\pi_{i} i=1,2,3$, we could study and combine several scenarios in order to see the impact and the effect of each one of these controls on the reduction of the rumor spread. The numerical resolution of the system with difference equations as well as the numerical simulations enabled us to compare and see the difference between each scenario in a concrete way. Numerical results prove the effectiveness of our strategy. The purpose of this work is achieved, and we have proved the effectiveness of our 
strategy and its importance in fighting the spread of any rumor throughout any social network.

\section{Data Availability}

The disciplinary data used to support the findings of this study have been deposited in the Network Repository (http://www.networkrepository.com).

\section{Conflicts of Interest}

The authors declare that they have no conflicts of interest.

\section{Acknowledgments}

Research reported in this paper was supported by the Moroccan Systems Theory Network.

\section{References}

[1] L. J. Sabato, K. Kondik, and G. Skelley, "Republicans 2016: what to do with the Donald?," 2015, http://centerforpolitics. org/crystalball/articles/republicans-2016-what-to-do-withthe-donald/.

[2] J. Fromm, S. Melzer, B. Ross, and S. Stieglitz, Trump versus Clinton-Twitter Communication during the US Primaries, Palgrave Macmillan, London, UK, 2016.

[3] J. C. Lee and K. Quealy, "The 337 people, places and things donald trump has insulted on twitter: a complete list," 2016, https://www.nytimes.com/interactive/2016/01/28/upshot/ donald-trump-twitter-insults.html.

[4] L. Cazzoli, R. Sharma, M. Treccani, and F. Lillo, "A large scale study to understand the relation between twitter and financial market," in Proceedings of the 2016 Third European Network Intelligence Conference, IEEE, Wroclaw, Poland, September 2016.

[5] A. N. Vilas, R. P. D. Redondo, and A. L. Garcia, "The irruption of cryptocurrencies into Twitter cashtags: a classifying solution," IEEE Access, vol. 8, pp. 32698-32713, 2020.

[6] L. Zhu, H. Zhao, and H. Wang, "Complex dynamic behavior of a rumor propagation model with spatial-temporal diffusion terms," Information Sciences, vol. 349-350, pp. 119-136, 2016.

[7] J. Ma, D. Li, and Z. Tian, "Rumor spreading in online social networks by considering the bipolar social reinforcement," Physica A: Statistical Mechanics and Its Applications, vol. 447, pp. 108-115, 2016.

[8] Q. Liu, T. Li, and M. Sun, "The analysis of an SEIR rumor propagation model on heterogeneous network," Physica A: Statistical Mechanics and Its Applications, vol. 469, pp. 372380, 2017.

[9] W. Huang, "On rumour spreading with skepticism and denial,” Technical Report, 2011.

[10] RE. Bartholomew and P. Hassall, A Colorful History of Popular Delusions, Prometheus Books, New York, NY, USA, 2015.

[11] W. O. Kermack and A. G. McKendrick, "A contribution to the mathematical theory of epidemics," Proceedings of the Royal Society of London Series A, vol. 115, no. 772, pp. 700-721, 1927.

[12] W. Goffman and V. A. Newill, "Generalisation of epidemic theory an application to the transmission of ideas," Nature, vol. 204, no. 4955, pp. 225-228, 1964.

[13] D. J. Daley and D. G. Kendall, "Epidemics and rumours," Nature, vol. 204, no. 4963, p. 1118, 1964.
[14] L. M. A. Bettencourt, A. Cintrón-Arias, D. I. Kaiser, and C. Castillo-Chávez, "The power of a good idea: quantitative modeling of the spread of ideas from epidemiological models," Physica A: Statistical Mechanics and Its Applications, vol. 364, pp. 513-536, 2006.

[15] H. Laarabi, A. Abta, M. Rachik, and J. Bouyaghroumni, "Stability analysis of a delayed rumor propagation model," Differential Equations and Dynamical Systems, vol. 24, no. 4, pp. 407-415, 2016.

[16] J. Jia and W. Wu, "A rumor transmission model with incubation in social networks," Physica A: Statistical Mechanics and Its Applications, vol. 491, pp. 453-462, 2018.

[17] C. Wang, Z. X. Tan, Y. Ye, L. Wang, K. H. Cheong, and N.-G. Xie, "A rumor spreading model based on information entropy," Scientific Reports, vol. 7, no. 1, 2017.

[18] C. Wang, J. M. Koh, K. H. Cheong, and N.-G. Xie, "Progressive information polarization in a complex-network entropic social dynamics model," IEEE Access, vol. 7, pp. 35394-35404, 2019.

[19] Z. X. Tan and K. H. Cheong, "Cross-issue solidarity and truth convergence in opinion dynamics," Journal of Physics A: Mathematical and Theoretical, vol. 51, no. 35, Article ID 355101, 2018.

[20] Y. Hu, Q. Pan, W. Hou, and M. He, "Rumor spreading model with the different attitudes towards rumors," Physica A: Statistical Mechanics and Its Applications, vol. 502, pp. 331344, 2018.

[21] S. Dong, F.-H. Fan, and Y.-C. Huang, "Studies on the population dynamics of a rumor-spreading model in online social networks," Physica A: Statistical Mechanics and Its Applications, vol. 492, pp. 10-20, 2018.

[22] A. Guille, H. Hacid, C. Favre, and D. A. Zighed, "Information diffusion in online social networks," ACM Sigmod Record, vol. 42, no. 2, pp. 17-28, 2013.

[23] O. Balatif, A. Labzai, and M. Rachik, "A discrete mathematical modeling and optimal control of the electoral behavior with regard to a political party," Discrete Dynamics in Nature and Society, vol. 2018, Article ID 9649014, 14 pages, 2018.

[24] A. Labzai, O. Balatif, and M. Rachik, "Optimal control strategy for a discrete time smoking model with specific saturated incidence rate," Discrete Dynamics in Nature and Society, vol. 2018, Article ID 5949303, 10 pages, 2018.

[25] A. Kouidere, O. Balatif, H. Ferjouchia, A. Boutayeb, and M. Rachik, "Optimal control strategy for a discrete time to the dynamics of a population of diabetics with highlighting the impact of living environment," Discrete Dynamics in Nature and Society, vol. 2019, Article ID 6342169, 8 pages, 2019.

[26] P. Georgescu and Y. H. Hsieh, "Global dynamics for a predator-prey model with stage structure for predator," SIAM Journal on Applied Mathematics, vol. 67, no. 5, pp. 379-1395, 2007.

[27] C. H. Jia and D. X. Feng, "An optimal control problem of a coupled nonlinear parabolic population system," Acta Mathematicae Applicatae Sinica, English Series, vol. 23, no. 3, pp. 377-388, 2007.

[28] A. El Alami Laaroussi and M. Rachik, "On the regional control of a reaction-diffusion system SIR," Bulletin of Mathematical Biology, vol. 82, no. 1, 2019.

[29] R. Ghazzali, A. E. A. Laaroussi, A. El Bhih, and M. Rachik, "On the control of a reaction-diffusion system: a class of SIR distributed parameter systems," International Journal of Dynamics and Control, vol. 7, no. 3, pp. 1021-1034, 2019.

[30] B. R. Soukaina, G. Rachid, A. El Bhih, and R. Mostafa, "Optimal control problem of a quarantine model in multi 
region with spatial dynamics," Communications in Mathematical Biology and Neuroscience, vol. 10, 2020.

[31] L. S. Pontryagin, V. G. Boltyanskii, R. V. Gamkrelidze, and E. Mishchenko, The Mathematical Theory of Optimal Processes (International Series of Monographs in Pure and Applied Mathematics), Interscience, New York, NY, USA, 1962.

[32] V. Guibout and A. M. Bloch, "A discrete maximum principle for solving optimal control problems," in Proceedings of the 2004 43rd IEEE Conference on Decision and Control (CDC), vol. 2, IEEE, Nassau, Bahamas, pp. 1806-1811, December 2004.

[33] W. Ding, R. Hendon, B. Cathey, E. Lancaster, and R. Germick, "Discrete time optimal control applied to pest control problems," Involve, A Journal of Mathematics, vol. 7, no. 4, pp. 479-489, 2014. 